\title{
Synthesis and characterization of pure and Co-doped gallium nitride nanocrystals
}

\author{
V. Ganesh $\cdot$ S. Suresh $\cdot$ E. Celasco • \\ K. Baskar
}

Received: 24 July 2011 / Accepted: 27 December 2011/Published online: 15 January 2012

(C) The Author(s) 2012. This article is published with open access at Springerlink.com

\begin{abstract}
Pure and Co-doped gallium nitride (GaN) (5 and $8 \mathrm{~mol} \%$ ) nanocrystals were synthesized by nitridation of Ga-EDTA. $\mathrm{NH}_{4}$ and Co-Ga-EDTA. $\mathrm{NH}_{4}$ complexes. Pure and $5 \mathrm{~mol} \%$ cobalt-doped GaN did not show any impurity phases in the X-ray diffraction and Raman analysis, whereas $8 \mathrm{~mol} \%$ Co-doped GaN show secondary phase formation. Transmission electron microscopic study revealed the broad crystal size distribution ranging from 10 to $140 \mathrm{~nm}$. Cathodoluminiscence spectra measured at $20 \mathrm{~K}$ shows the suppression of $\mathrm{GaN}$ band edge emission with increasing Co concentration. Magnetic measurements of Co-doped GaN revealed a ferromagnetic behavior up to room temperature. The saturation magnetization value increases with increasing impurity phase separation. X-ray photoelectron spectroscopy revealed evidence on the oxidation states of cobalt and excludes the possibility of Co clusters in the doped samples.
\end{abstract}

Keywords Semiconductors · Dilute magnetic semiconductors - Nanocrystals · X-ray diffraction · Raman spectroscopy · XPS · TEM · SQUID

\section{Introduction}

Gallium nitride is III-V semiconductor with a direct band gap of $3.4 \mathrm{eV}$ at room temperature. It is an ideal material for $\mathrm{UV}$ or

V. Ganesh $(\bowtie) \cdot$ S. Suresh $\cdot$ K. Baskar

Crystal Growth Centre, Anna University Chennai,

Chennai 600 025, India

e-mail: 80.vganesh@gmail.com

E. Celasco

Materials Science and Chemical Engineering Department, Politecnico di Torino, C.so Duca degli Abruzzi 24, 10129 Torino, Italy blue emitters, detectors, high-speed field-effect transistors, and high-temperature/high-power electronic devices. In the past few years, extensive research has been focused on the study of hexagonal GaN nanostuctures, such as nanowires (nanorods) (Duan and Lieber 2000; Li et al. 2000a; Chen et al. 2000; Chen et al. 2001), nanotubes (Li et al. 2001a), nanoribbons (Li et al. 2000b), nanorings (Li et al. 2001b), and so forth. The study on nano-GaN continues at a rapid pace to understand the fundamental optical properties. Semiconducting materials in which a fraction of the host cations can be replaced by magnetic ions are known as diluted magnetic semiconductors (DMS), or sometimes referred to as semimagnetic semiconductors. To have practical applications in spintronics devices, these DMS materials must exhibit ferromagnetism, with a critical temperature above room temperature [Curie temperature $\left.\left(T_{\mathrm{c}}\right)\right]$. Mn-doped $\mathrm{GaN}(\mathrm{GaMnN})$ was predicted by Dietl et al. (2000) to be ferromagnetic with a high Curie temperature and therefore could have a possible use in spintronics. Several experimental studies were reported on the magnetic properties of GaMnN (Pearton et al. 2004; Liu et al. 2005; Reed et al. 2001; Thaler et al. 2002; Overberg et al. 2001). Several groups have reported Curie temperatures near or above the room temperature for Mn-doped GaN (Reed et al. 2001; Theodoropoulou et al. 2001). There are not much detailed study on the synthesis of cobalt (Co)-doped GaN nanocrystals. Previous reports on Co-doped GaN have shown ferromagnetic (hysteresis) behavior at $5 \mathrm{~K}$ and below room temperature (Sawahata et al. 2005; Dhara et al. 2006; Kim et al. 2006a). The present article shows the synthesis of pure and cobalt (Co)-doped $\mathrm{GaN}$ nanocrystals by nitridation of Ga-EDTA and Co-doped Ga-EDTA complexes and the existence of ferromagnetic behavior at room temperature. Furthermore, the structure, morphology and optical properties of the pure and Co-doped GaN nanocrystals were studied and discussed. 


\section{Experiment}

Ga-EDTA. $\mathrm{NH}_{4}$ complex was prepared from the mixture of gallium trichloride $\left(\mathrm{GaCl}_{3}\right)$, ethylene diamine tetra acetic acid (EDTA) and liquid ammonia in an aqueous solution at a $\mathrm{pH}$ of 9. The solution was stirred for $6 \mathrm{~h}$ and dried in an oven at $343 \mathrm{~K}$ to yield a Ga-EDTA. $\mathrm{NH}_{4}$ complex. Complex was loaded in to the quartz reactor which is purged with nitrogen till the synthesis temperature of $1,173 \mathrm{~K}$. Ammonia was introduced at the synthesis temperature. The synthesis was carried out for a reaction period of $8 \mathrm{~h}$ at $1,173 \mathrm{~K}$ to obtain pure $\mathrm{GaN}$ nanocrystals. After the synthesis, the flow of $\mathrm{NH}_{3}$ is stopped when the temperature drops down to $773 \mathrm{~K}$; however, $\mathrm{N}_{2}$ purging continued until the room temperature. Cobalt chloride was used as a source of cobalt doping in GaN. 5 and $8 \mathrm{~mol} \%$ of $\mathrm{CoCl}_{2}$ was added to the Ga-EDTA complex and nitridated as mentioned above at $1,173 \mathrm{~K}$ for a period of $8 \mathrm{~h}$.

The synthesized samples were analyzed using various techniques. Powder X-ray diffraction patterns (XRD) of GaN crystals were recorded using $\mathrm{Cu}-\mathrm{K} \alpha$ radiation of wavelength $1.5418 \AA$ at a scan speed of 1 degree per minute. The morphologies of $\mathrm{GaN}$ were studied using transmission electron microscopy (TEM) and the dimension of the crystals was estimated from the TEM data using the standard software (Image-J). The optical properties of the pure and Co-doped $\mathrm{GaN}$ nanocrystals were analyzed by cathodoluminiscence (CL) spectroscopy at a temperature of $20 \mathrm{~K}$. Samples were excited with an electron beam of energy $2 \mathrm{keV}$, at a current of 6 nA. The Renishaw Ramascope system-model 1,000 with an excitation of $\mathrm{He}-\mathrm{Ne}$ laser $(514 \mathrm{~nm})$ was used for Raman experiments. Magnetic measurements of Co-doped $\mathrm{GaN}$ were carried out using a MPMS-SQUID (Superconducting Quantum Interference Device). Magnetization versus magnetic field ( $M-H$ curve) was measured for doped samples between $-20,000$ and 20,000 Oe. The temperature versus magnetization measurements were carried out for doped samples between 10 and $300 \mathrm{~K}$ at applied field of $1 \mathrm{KOe}$. The X-ray photoelectron spectroscopy (XPS) measurements were carried out using an electron spectrometer PHI 5000 Versa Probe Scanning ESCA Microprobe, with a monochromatic X-ray beam Al $\mathrm{K} \alpha(1,486.6 \mathrm{eV})$ source. The X-ray diameter employed had $100 \mu \mathrm{m}$ spot on a square area of $400 \times 400 \mu \mathrm{m}$; this area was chosen to have good average information of powder behavior. The sensitivity range of XPS instrument is $0.1-1$ at\%.

\section{Results and discussion}

X-ray diffraction

Figure 1 shows XRD pattern of pure and Co-doped GaN nanocrystals. Pure and 5 mol\% Co-doped GaN spectrums

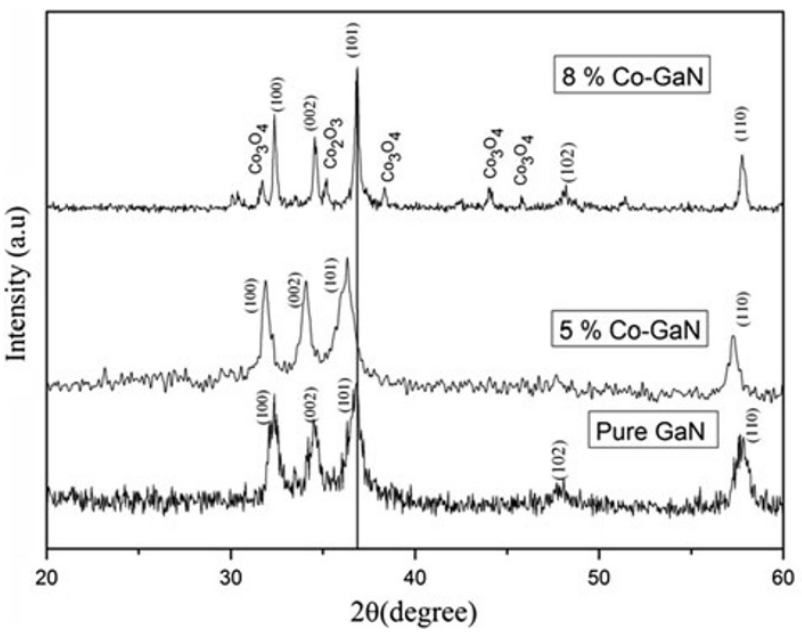

Fig. 1 XRD patterns of pure and doped GaN nanocrystals

show diffraction peaks associated with the single phase wurtzite structure of GaN. Although XRD spectrum of 8 mol\% Co-doped GaN shows secondary phase and assigned as cobalt oxide $\left(\mathrm{Co}_{3} \mathrm{O}_{4}\right)$. The lattice parameters were evaluated from the XRD data for pure $\mathrm{GaN}$ is $a=3.19 \AA, c=5.19 \AA$, respectively, which are in good agreement with the reported values (Iwata et al. 1996). The lattice parameters for $5 \mathrm{~mol} \% \mathrm{Co}$-doped $\mathrm{GaN}$ were calculated as $a=3.23 \AA, c=5.25 \AA$. The XRD peaks of 5 mol\% Co-doped GaN shift towards a lower angle implying an increase in lattice parameters as compared to pure $\mathrm{GaN}$. This is because of the larger ionic radii of $\mathrm{Co}^{3+}$ $(0.54 \AA)$ as compared to that of $\mathrm{Ga}^{3+}(0.47 \AA)$. An increase in lattice parameters confirms that Co ions are substituted in the Ga sub-lattice generating tensile strain, the changes in lattice parameters are similar to the previous reports (Ramachandran et al. 2004; Ueda et al. 2001). Significant change in lattice parameter was not observed in the $8 \mathrm{~mol} \%$ of Co-doped GaN due to the formation of secondary phase.

\section{Raman spectroscopy}

Raman spectra of pure and Co-doped GaN nanocrystals are shown in Fig. 2. The undoped GaN nanocrystals exhibit five phonon modes observed at 419, 535, 556, 568 and $728 \mathrm{~cm}^{-1}$. The $419 \mathrm{~cm}^{-1}$ phonon mode correspond to the acoustic overtone of $\mathrm{GaN}$, and the other four phonon modes corresponds to $\mathrm{A}_{1}$ (TO), $\mathrm{E}_{1}$ (TO), $\mathrm{E}_{2}$ (High) and $\mathrm{A}_{1}$ (LO) modes, respectively (Perlin et al. 1992; Harima 2002). For Co-doped GaN samples, all the modes broaden and shift to the lower frequency as compared to pure GaN. The nonpolar $\mathrm{E}_{2}$ (High) mode is a good choice to understand the disorder-induced phenomena in GaN upon doping. Its pronounced red shift, broadening, and weakening for the doped GaN samples, as compared to pure GaN 


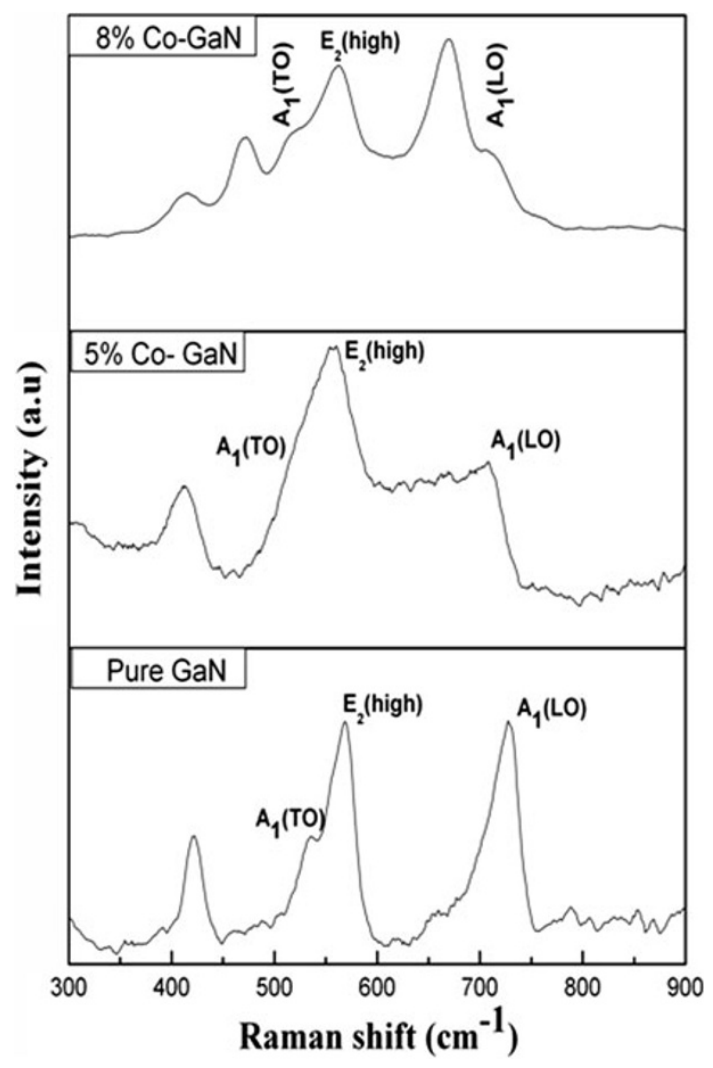

Fig. 2 Raman spectra of pure and Co-doped GaN nanocrystals

shows that structural defects and local lattice distortions are high in these samples induced by doping. This behavior was observed in Co-doped $\mathrm{ZnO}$ by previous authors (Hasuike et al. 2007; Wang et al. 2007). The $A_{1}$ (LO) phonons signal sharply peaked for pure sample, whereas the $\mathrm{A}_{1}(\mathrm{LO})$ phonon mode becomes strongly asymmetric in $5 \mathrm{~mol} \%$ Co-doped sample. The strong asymmetric broadening is due to the lattice distortion and strain induced by the doping. For higher doping levels, the long range lattice ordering is seriously deteriorated. The additional phonon modes are observed for $8 \mathrm{~mol} \%$ Co-doped $\mathrm{GaN}$ at 472 and $670 \mathrm{~cm}^{-1}$ are assigned to $\mathrm{E}_{\mathrm{g}}$ and $\mathrm{A}_{1 \mathrm{~g}}$ modes of $\mathrm{Co}_{3} \mathrm{O}_{4}$ phase (Wang et al. 2006, 2008), whereas for $5 \mathrm{~mol} \% \mathrm{Co}-$ doped $\mathrm{GaN}$, no secondary phase phonon modes were observed. The Raman results are well consistent with the XRD results.

Transmission electron microscopy

Figure $3 a-c$ shows a TEM micrograph for the pure and Co-doped GaN sample. It has been clearly observed that the average crystal size increases in nanometric regime with the increase in dopant concentration. Figure $3 \mathrm{~d}$ shows the particle size distribution of pure and cobalt-doped $\mathrm{GaN}$ nanocrystals. The diameter range is between 10 and
$140 \mathrm{~nm}$ for pure and doped samples. The average diameter of pure GaN nanocrystals is $20 \mathrm{~nm}$. In $5 \mathrm{~mol} \%$ Co-doped $\mathrm{GaN}$ nanocrystals, the increase in size is due to the lattice expansion because of the larger ionic radii of Co as compared to $\mathrm{Ga}$ and similar behavior was reported earlier by Mandal et al. (2006). Although in 8 mol\% Co-doped GaN nanocrystals, the size variation is larger when compared with pure $\mathrm{GaN}$ and this may be due to the agglomeration of $\mathrm{Co}_{3} \mathrm{O}_{4}$ phases on the surface of $\mathrm{GaN}$ nanocrystals, which may also counted during particle size analysis.

\section{Cathodoluminescence}

Pure and 5 mol\% Co-doped samples exhibit the bandedge emission of $\mathrm{GaN}$ at $3.49 \mathrm{eV}$ is shown in Fig. 4. The $\mathrm{CL}$ spectrum shows the progressive reduction in intensity of band-edge luminescence with increasing Co concentration. The decrease in the intensity of band-edge luminescence with Co doping seems to be due to the presence of traps or defect states in the band gap originated by the doping of Co (Yang et al. 2008; Lvill et al. 2008). The band at $3.263 \mathrm{eV}$ for undoped and the bands at $3.21 \mathrm{eV}$ for Co-doped $\mathrm{GaN}$ were observed, these emissions were also identified by previous authors and assigned as donor-acceptor pair emission (DAP) for doped and undoped GaN (Lagerstedt and Monemar 1974; Loan et al. 2009). For $8 \mathrm{~mol} \%$ Co-doped GaN, the band-edge emission becomes weaker as compared to the DAP emission. Thus, decrease in band-edge emission can be attributed to an increase in impurity levels or traps in the Co-doped GaN samples with increasing Co concentration. CL spectrum shows tails toward longer and shorter wavelengths. This implies the existence of (1) small GaN nanoparticles that exhibit the quantum confinement effect and (2) crystallized (bigger in size) $\mathrm{GaN}$ emits light at the band-edge region.

\section{Magnetic measurements}

Figure 5a-c shows the variation of magnetization $(M)$ with magnetic field $(H)$ measured at 10 and $300 \mathrm{~K}$ for Co-doped samples and at $300 \mathrm{~K}$ for pure samples. Pure $\mathrm{GaN}$ exhibits diamagnetic behavior as shown in Fig. 5a, whereas 5 and 8 mol\% Co-doped GaN exhibit a hysteresis loop at both the temperatures which is shown in Fig. 5b, c, respectively. The measurements were made as such (in powder form) without substrates. The ferromagnetism at 10 and $300 \mathrm{~K}$ is clearly shown by the coercivity and remanence suggesting that the Curie temperature $\left(T_{\mathrm{c}}\right)$ is at least $300 \mathrm{~K}$. The magnetization values are well saturated for both the samples indicating room temperature ferromagnetism. The observed magnetic behavior may be attributed to the exchange interaction between the localized magnetic dipole moments of the magnetic ions (the localized $d$-spins 

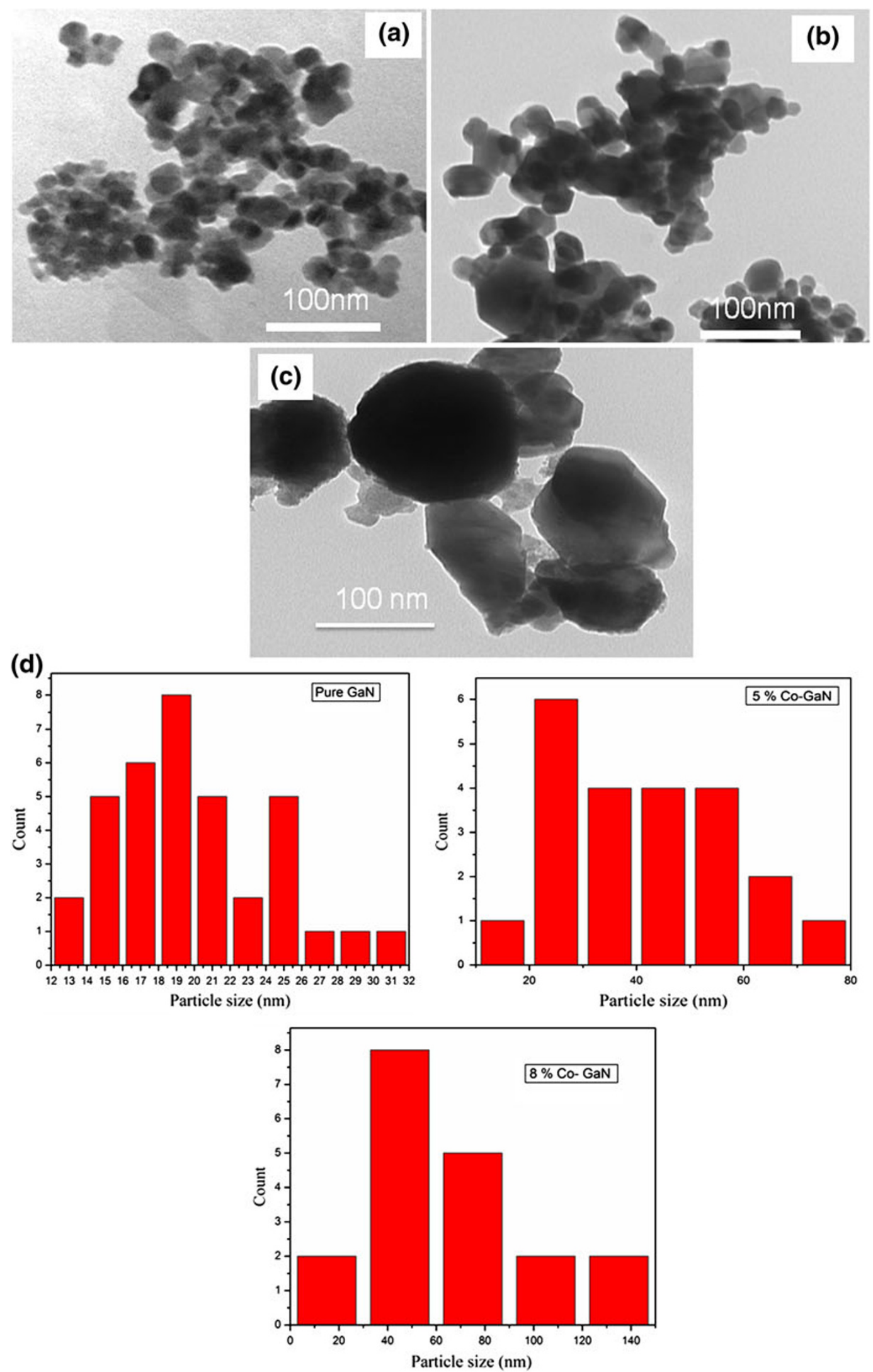

Fig. 3 TEM images of pure and Co-doped GaN nanocrystals. a Pure GaN, b 5\% Co-doped GaN and c 8\% Co-doped GaN. d Particle size distribution of pure and Co-doped $\mathrm{GaN}$ nanocrystals 


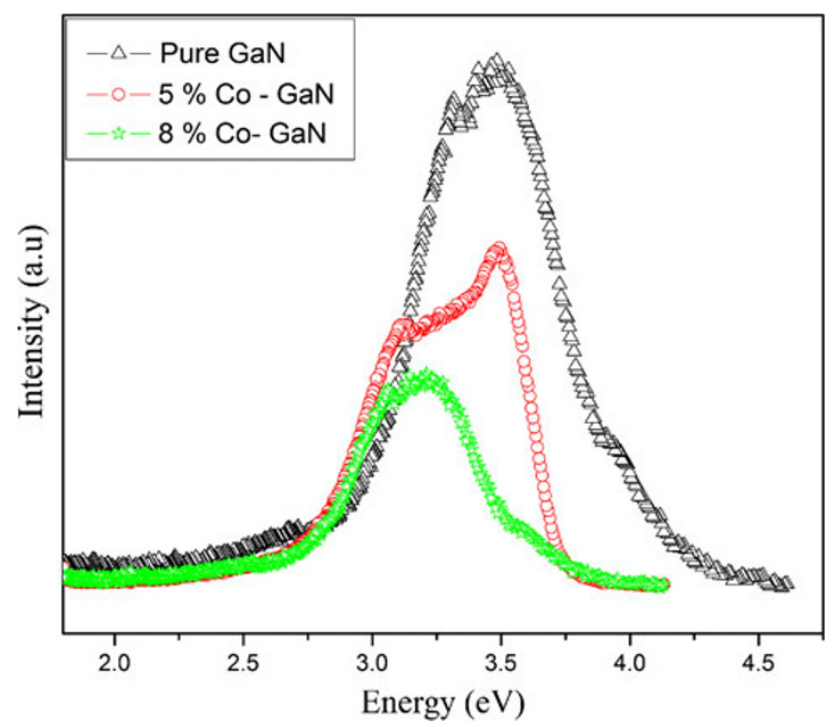

Fig. 4 CL spectra of pure and Co-doped GaN nanocrystals measured at $20 \mathrm{~K}$
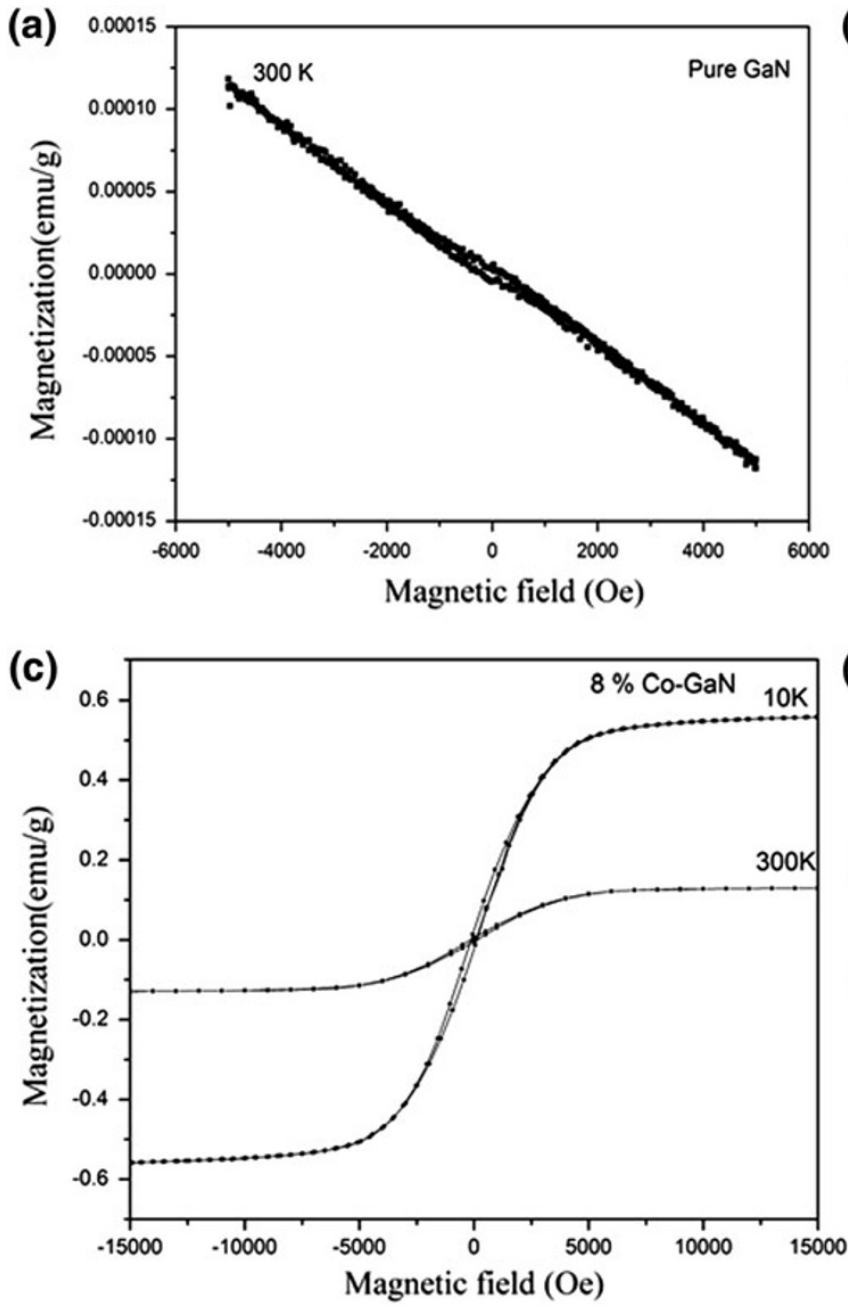

of Co ions) and the free delocalized charge of current carriers (holes or electrons from the valence band).

The saturation magnetization $\left(M_{\mathrm{s}}\right)$ values increase with increasing Co concentration. The $M$ versus $T$ curve was recorded for $5 \mathrm{~mol} \%$ Co-doped $\mathrm{GaN}$ nanocrystals by measuring the magnetization difference of field cooled (FC) and zero-field-cooled (ZFC) conditions with an applied field of 1,000 Oe as shown in Fig. 5d. The difference $\Delta M=M_{\mathrm{FC}}-M_{\mathrm{ZFC}}$ subtraction is particularly effective when there are small amounts of ferromagnetic material in the presence of a large diamagnetic and/or paramagnetic background. This subtraction simultaneously indicates the presence of hysteresis if the difference is nonzero. The data indicate that the ferromagnetism persists to $\sim 300 \mathrm{~K}$ for both the samples (Han et al. 2005). The $M$ versus $T$ curve was also recorded for $8 \mathrm{~mol} \%$ Co-doped $\mathrm{GaN}$ nanocrystals, which are not shown here because of the observation of impurity phases by XRD and Raman analysis. In $8 \mathrm{~mol} \%$ Co-doped sample, the increase in
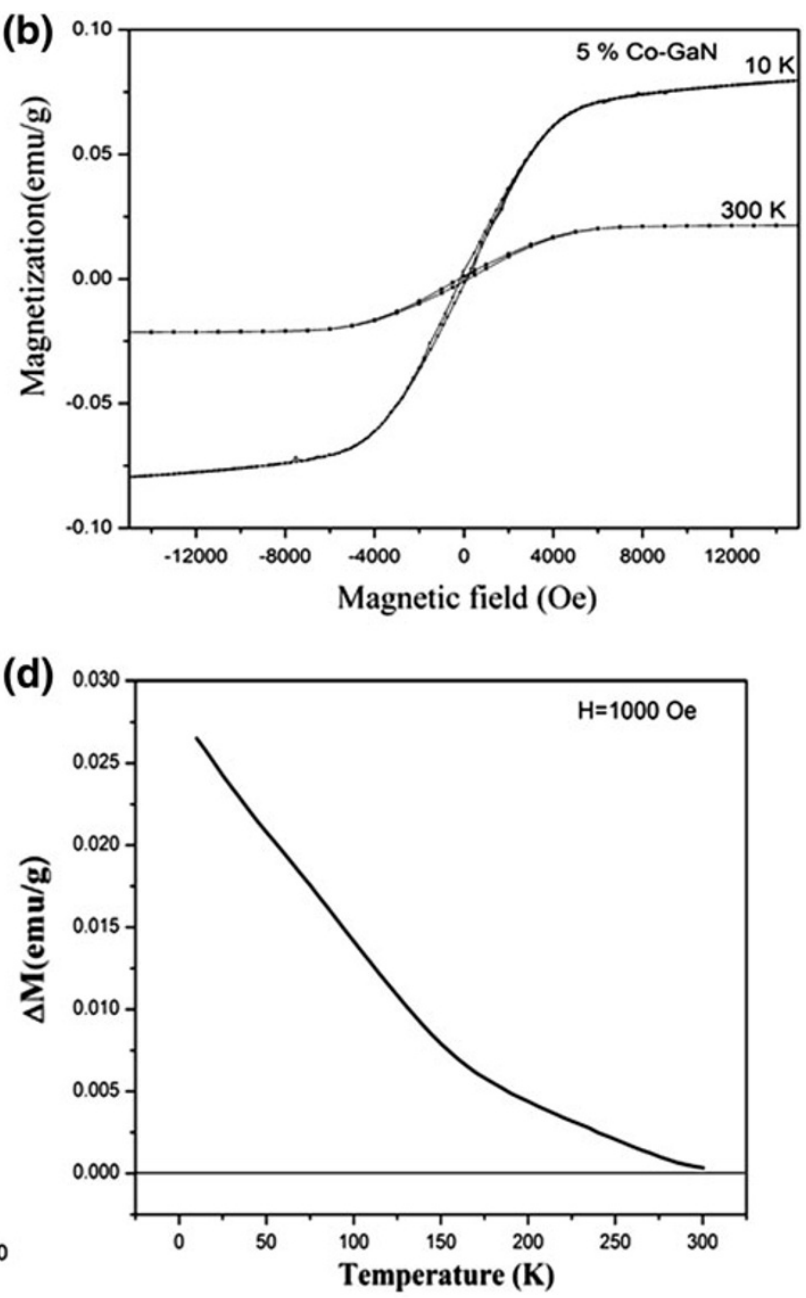

Fig. 5 a-c Magnetization loops for pure, 5 and $8 \%$ of Co-doped GaN, c temperature-dependent $\Delta M=M_{\mathrm{FC}}-M_{\mathrm{ZFC}}$ measured with $H=\mathrm{I}$ kOe for $5 \mathrm{~mol} \%$ of the Co-doped GaN nanocrystals 

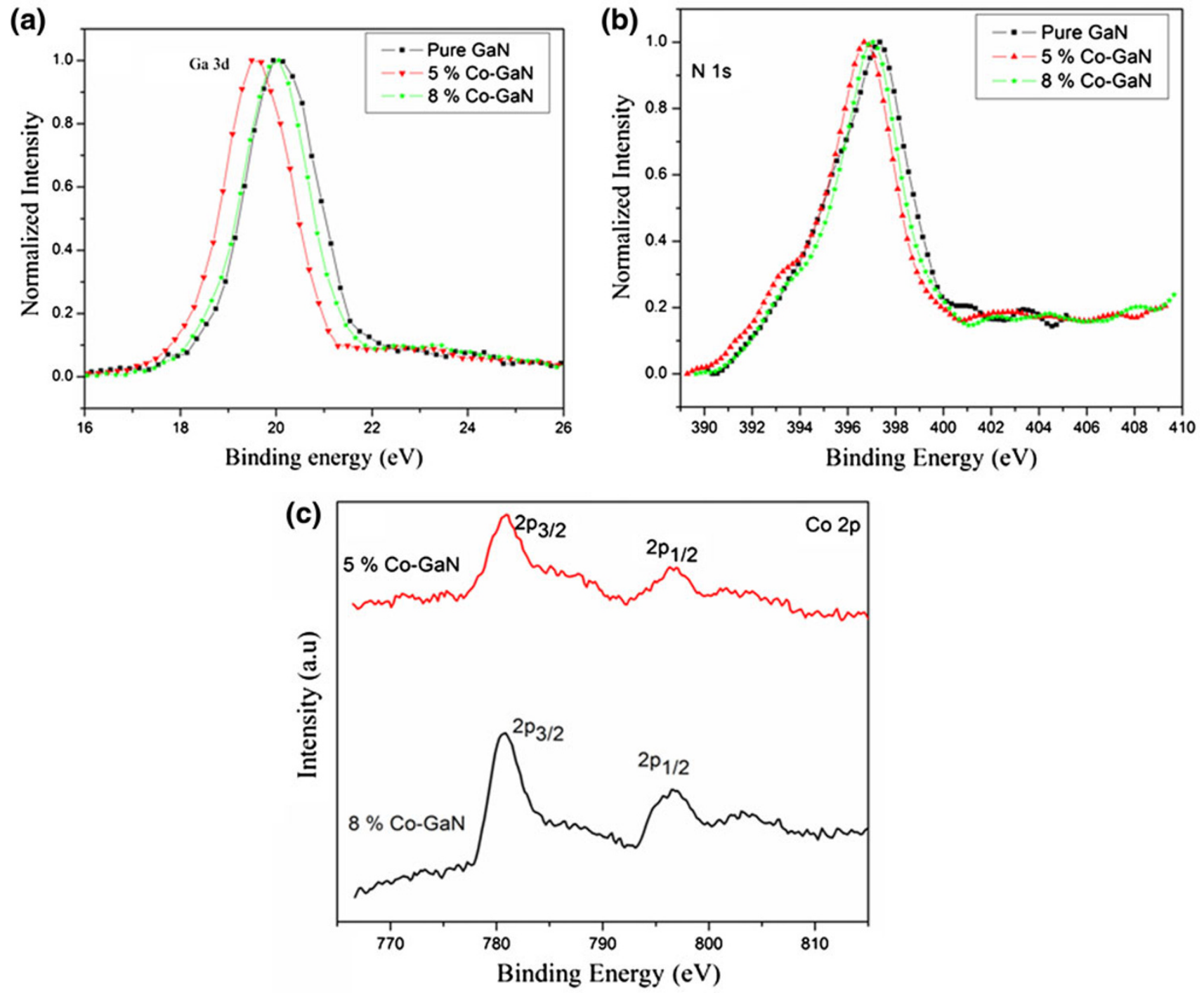

Fig. 6 XPS spectra of pure and Co-doped GaN nanocrystals

saturation magnetization is due to the Co containing impurity phases, but in $5 \mathrm{~mol} \%$ Co-doped sample, it may be due to diluted magnetic behavior of GaN.

\section{X-ray photoelectron spectroscopy}

To understand the origin of magnetic property in the Co-doped samples, the XPS analysis was performed for the synthesized samples. The spectra were referred to $\mathrm{C} 1 \mathrm{~s}$ photoelectron peak at $284.8 \mathrm{eV}$. Figure $6 \mathrm{a}-\mathrm{c}$ shows the XPS spectra of Ga 3d, N 1s and Co 2p, respectively. XPS measurements of 5 and $8 \mathrm{~mol} \%$ doped samples show that the energy difference between the Co $2 \mathrm{p}_{3 / 2}$ and Co $2 \mathrm{p}_{1 / 2}$ core level is 15.3 and $15.9 \mathrm{eV}$, respectively. If the cobalt atoms in the sample were all surrounded by oxygen, the energy difference between Co $2 \mathrm{p}_{3 / 2}$ and Co $2 \mathrm{p}_{1 / 2}$ peak should be $15.5 \mathrm{eV}$ which confirm the Co is surrounded by oxygen (Martínez et al. 2005). If the cobalt atoms in the sample exist as metal clusters, the energy difference should be $14.8 \mathrm{eV}$ (Park et al. 2004). From these results, the possibility of metallic Co clusters in the doped samples is very less. The difference in binding energy shifts towards the Co-O bond with increasing Co concentration; these results are also supported by XRD and Raman analysis which shows that the formation of cobalt oxide at higher cobalt concentrations. The Ga $3 \mathrm{~d}$ spectrum of the pure sample consists of $\mathrm{Ga}-\mathrm{N}$ and $\mathrm{Ga}-\mathrm{O}$ bonds whereas the Co-doped samples shows only a $\mathrm{Ga}-\mathrm{N}$ bond and the binding energies of $\mathrm{Ga}-\mathrm{N}$ is closely matched to the reported values (Lee et al. 2002; Kim et al. 2006b; Xiao et al. 2005). The $\mathrm{N} 1 \mathrm{~s}$ spectrum shows the typical bond of nitrogen with $\mathrm{Ga}$ and also at the lower binding energy 
side observed the overlapping of Ga LMM Auger peak with $\mathrm{N}$.

\section{Conclusion}

Pure and Co-doped GaN nanocrystals were prepared by nitridation of Ga-EDTA and Co-doped Ga-EDTA complexes at $1,173 \mathrm{~K}$. The crystalline structure of pure and Co-doped GaN nanocrystals were confirmed by XRD and Raman analysis. The shift in the XRD and Raman peaks proved the incorporation of $\mathrm{Co}$ into the GaN lattice. The TEM micrographs showed a broad crystal size distribution ranging from 10 to $140 \mathrm{~nm}$ of pure and doped $\mathrm{GaN}$ nanocrystals. The decrease in band-edge emission with increasing dopant concentration was observed by $\mathrm{Cl}$ spectrum. The hysteresis curves measured at 10 and $300 \mathrm{~K}$, and the temperature-dependent curves $\Delta M=M_{\mathrm{FC}}-M_{\mathrm{ZFC}}$ with $H=1 \mathrm{kOe}$, provide evidence for the room temperature ferromagnetism in Co-doped GaN nanocrystals.

Acknowledgments The authors acknowledge the CSIR, Government of India, for the award of Senior Research fellowship and for financial support. The authors also acknowledge Dr. A. Banerjee, UGC-CSR Indore, for his help in magnetic measurements.

Open Access This article is distributed under the terms of the Creative Commons Attribution License which permits any use, distribution and reproduction in any medium, provided the original author(s) and source are credited.

\section{References}

Chen XL, Li JY, Cao YG, Lan YC, Li H, He M, Wang CY, Zhang Z, Qiao ZY (2000) Straight and smooth GaN nanowires. Adv Mater 12:1432-1434

Chen CC et al (2001) Catalytic growth and characterization of gallium nitride nanowires. J Am Chem Soc 123:2791-2798

Dhara S, Sundaravel B, Nair KGM, Kesavamoorthy R, Valsakumar MC (2006) Ferromagnetism in cobalt-doped n-GaN. Appl Phys Lett 88:173110-173113

Dietl T, Ohno H, Matsukara F, Cibert J, Ferrand D (2000) Zener model description of ferromagnetism in zinc-blende magnetic semiconductors. Science 287:1019-1022

Duan XF, Lieber CM (2000) Laser-assisted catalytic growth of single crystal GaN nanowires. J Am Chem Soc 122:188-189

Han DS, Park J, Rhie KW, Kim S, Chang J (2005) Ferromagnetic Mn-doped GaN nanowires. Appl Phys Lett 86:032506-3

Harima H (2002) Properties of GaN and related compounds studied by means of Raman scattering. J Phys Condens Matter 14:R967R993

Hasuike N, Deguchi R, Katoh H, Kisoda K, Nishio K, Isshiki T, Harima $\mathrm{H}$ (2007) Structural properties of nanometer-sized $\mathrm{ZnO}$ crystals doped with Co. J Phys Condens Matter 19:365223-365228

Iwata K, Asahi H, Yu SJ, Asami K, Fujita H, Fushida M, Gonda S (1996) High quality GaN growth on (0001) sapphire by ionremoved electron cyclotron resonance molecular beam epitaxy and first observation of $(2 \times 2)$ and $(4 \times 4)$ reflection high energy electron diffraction patterns. Jpn J Appl Phys 35:L289L292

Kim W et al (2006a) Magnetic and structural properties of Co ion-implanted GaN. IEEE Trans Nanotechnol 5:149-151

Kim W, Kang HJ, Oh SK, Shin SW, Lee JH, Song JH, Noh SK, OH SJ, Kim SJ, Kim CS (2006b) Co ion-implanted GaN and its magnetic properties. J Magn 11:16-19

Lagerstedt O, Monemar B (1974) Luminescence in epitaxial GaN:Cd. J Appl Phys 45:2266-2272

Lee HJ, Jeong SY, Cho CR, Park CH (2002) Study of diluted magnetic semiconductor: Co-doped $\mathrm{ZnO}$. Appl Phys Lett 81:4020-4022

Li JY, Chen XL, Qiao ZY, Cao YG, Lan YC (2000a) Formation of GaN nanorods by a sublimation method. J Cryst Growth 213:408-410

Li JY, Qiao ZY, Chen XL, Cao YG, Lan YC, Wang CY (2000b) Morphologies of GaN one-dimensional materials. Appl Phys A Mater Sci Process 71:587-588

Li JY, Chen XL, Qiao ZY, Cao YG, Li H (2001a) Synthesis of GaN nanotubes. J Mater Sci Lett 20:1987-1988

Li J, Qiao Z, Chen X, Cao Y, He MJ (2001b) Gallium nitride nanoribbon rings. J Phys Condens Matter 13:L285-L290

Liu C, Yun F, Morkok H (2005) Ferromagnetism of ZnO and GaN: a review. J Mater Sci Mater Electron 16:555-597

Loan TT, Long NN, Ha LH (2009) Photoluminescence properties of Co-doped $\mathrm{ZnO}$ nanorods synthesized by hydrothermal method. J Phys D Appl Phys 42:065412-065417

Lvill M, Pearton SJ, Rawal S, Leu L, Sadik P (2008) Structure and magnetism of cobalt-doped $\mathrm{ZnO}$ thin films. New J Phys 10:065002-065021

Mandal SK, Das AK, Nath TK (2006) Microstructural and magnetic properties of $\mathrm{ZnO}: \mathrm{TM}(\mathrm{TM}=\mathrm{Co}, \mathrm{Mn})$ diluted magnetic semiconducting nanoparticles. J Appl Phys 100:104315-104318

Martínez B, Sandiumenge F, Balcells L, Arbiol J, Sibieude F, Monty C (2005) Structure and magnetic properties of Co-doped $\mathrm{ZnO}$ nanoparticles. Phys Rev B 72:165202-165208

Overberg ME, Abernathy CR, Pearton SJ, Theodoropoulou NA, McCarthy KT, Hebard AF (2001) Indication of ferromagnetism in molecular-beam-epitaxy-derived N-type GaMnN. Appl Phys Lett 79:1312-1314

Park JH, Kim MG, Jang HM, Ryu S, Kim YM (2004) Co-metal clustering as the origin of ferromagnetism in Co-doped $\mathrm{ZnO}$ thin films. Appl Phys Lett 84:1338-1340

Pearton SJ et al (2004) Wide bandgap GaN-based semiconductors for spintronics. J Phys Condens Matter 16:R209-R245

Perlin P, Carillon CJ, Itie JP, Miguel AS, Grzegory I, Polian A (1992) Raman scattering and X-ray-absorption spectroscopy in gallium nitride under high pressure. Phys Rev B 45:83-89

Ramachandran S, Tiwari A, Narayani J (2004) Origin of roomtemperature ferromagnetism in cobalt-doped $\mathrm{ZnO}$. J Electron Mater 33:1298-1302

Reed ML, El-Masry NA, Stadelmaier HH, Ritums MK, Reed MJ, Parker CA, Roberts JC, Bedair SM (2001) Room temperature ferromagnetic properties of $(\mathrm{Ga}, \mathrm{Mn}) \mathrm{N}$. Appl Phys Lett 79:3473-3475

Sawahata J, Bang H, Takiguchi M, Seo J, Yanagihara H, Kita E, Akimoto K (2005) Structural and magnetic properties of Co doped GaN. Phys Stat Sol (c) 2:2458-2462

Thaler GT et al (2002) Magnetic properties of n-GaMnN thin films. Appl Phys Lett 80:3964-3966

Theodoropoulou N, Hebard AF, Overberg ME, Abernathy CR, Pearton SJ, Chu S NG, Wilson RG (2001) Magnetic and structural properties of Mn-implanted GaN. Appl Phys Lett 78:3475

Ueda K, Tabata H, Kawai T (2001) Magnetic and electric properties of transition-metal-doped ZnO films. Appl Phys Lett 79:988-990

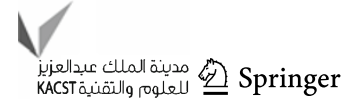


Wang XF, Xu JB, Zhang B, Yu HG, Wang J, Zhang X, Yu JG, Li Q (2006) Signature of intrinsic high-temperature ferromagnetism in cobalt-doped zinc oxide nanocrystals. Adv Mater 18:2476-2480

Wang X, Xu J, Yu X, Xue K (2007) Structural evidence of secondary phase segregation from the Raman vibrational modes in $\mathrm{Zn}_{1-}$ ${ }_{x} \mathrm{Co}_{x} \mathrm{O}(0<x<0.6)$. Appl Phys Lett 91:031908-3

Wang X, Zheng R, Liu Z, Ho-Pui H, Xu J, Ringer SP (2008) Structural, optical and magnetic properties of Co-doped $\mathrm{ZnO}$ nanorods with hidden secondary phases. Nanotechnology 19:455702-455708

Xiao HD et al (2005) Synthesis and structural properties of GaN particles from $\mathrm{GaO}_{2} \mathrm{H}$ powders. Diam Relat Mater 14:1730-1734

Yang XL, Chen ZT, Zhao LB, Zhu WX, Wang CD, Pei XD, Zhang GY (2008) Structural, optical and magnetic properties of Ga1 $-x \mathrm{Mn} x \mathrm{~N}$ films grown by MOCVD. J Phys D Appl Phys 41:245004-4 